\title{
Metabarcoding analysis of gut microbiota of healthy individuals reveals impact of probiotic and maltodextrin consumption
}

\author{
M. Calgaro ${ }^{1 \#}$, M. Pandolfo ${ }^{1 \#}$, E. Salvetti ${ }^{1,2^{*}}$, A. Marotta ${ }^{3}$, I. Larini ${ }^{1}$, M. Pane ${ }^{4}$, A. Amoruso ${ }^{4}$, A. Del Casale ${ }^{2}$, \\ N. Vitulo ${ }^{1 *}$, M. Fiorio ${ }^{3}$ and G.E. Felis ${ }^{1}$ \\ ${ }^{1}$ Department of Biotechnology, University of Verona, Verona, Strada le Grazie 15, 37134 Verona, Italy; ${ }^{2}$ Open Innovation \\ Department, Microbion SRL, via Monte Carega, 22, 37057 San Giovanni Lupatoto, Verona, Italy; ${ }^{3}$ Department of \\ Neurosciences, Biomedicine and Movement Sciences, University of Verona, Verona, Italy; ${ }^{4}$ Probiotical Research SRL, Via \\ Enrico Mattei 3, 28100 Novara, Italy; nicola.vitulo@univr.it; elisa.salvetti@univr.it; " these authors contributed equally to \\ this work
}

Received: 15 July 2020 / Accepted: 24 December 2020

(c) 2021 Wageningen Academic Publishers

OPEN ACCESS @(1)(2) RESEARCH ARTICLE

\begin{abstract}
In a previously published double-blind, placebo-controlled study, we showed that probiotics intake exerted a positive effect on sleep quality and a general improvement across time in different aspects of the profile of mood state, like sadness, anger, and fatigue in 33 healthy individuals. The present work investigates the impact of the probiotic product, constituted of Limosilactobacillus fermentum LF16, Lacticaseibacillus rhamnosus LR06, Lactiplantibacillus plantarum LP01 (all former members of Lactobacillus genus), and Bifidobacterium longum 04, on the gut microbiota composition of the same cohort through a metabarcoding analysis. Both the placebo and probiotic treatments had a significant impact on the microbiota composition. Statistical analysis showed that the microbiota of the individuals could be clustered into three groups, or bacteriotypes, at the baseline, and, inherently, bacterial compositions were linked to different responses to probiotic and placebo intakes. Interestingly, L. rhamnosus and L. fermentum were retrieved in the probiotic-treated cohort, while a bifidogenic effect of maltodextrin, used as placebo, was observed. The present study shed light on the importance of defining bacteriotypes to assess the impact of interventions on the gut microbiota and allowed to reveal microbial components which could be related to positive effects (i.e. sleep quality improvement) to be verified in further studies.
\end{abstract}

Keywords: gut microbiota, metabarcoding analysis, lactobacilli, Bifidobacterium, maltodextrin

\section{Introduction}

A great interest has emerged in the last years on the impact of the gut-brain axis in psychiatric disorders, pointing to stressed and unhealthy conditions of the microbial communities inside the human gut as possible causes for psychological diseases and conditions, such as depression, anxiety and schizophrenia (Bastiaansen et al., 2020; Kelly et al., 2019). It has been observed that the gut microbiota communicates with the brain, exerting effects over several neurobiological mechanisms and related systems; among these the hypothalamic-pituitary-adrenal axis, the immune system, the tryptophan metabolism and the production of various neuroactive compounds (Kelly et al., 2019).
For those reasons, the gut microbiota has become a new target to obtain antidepressant effects; remarkably, the diversity of studies performed and the functional redundancy of the microbiome make it difficult to understand if specific microbial components are more related than others to psychiatric symptoms (Cheung et al., 2019).

Since microbiota composition can be modified in a variety of ways, such as through the use of probiotics, prebiotics and dietary changes (Butler et al., 2019; Cheung et al., 2019), several clinical and translational studies have been published over the years, showing that the prolonged prebiotic and probiotic consumption can positively affect 
aspects of mood, anxiety, and cognition in both healthy individuals as well as in patients diagnosed with clinical psychiatric disorders (Butler et al., 2019; Kelly et al., 2019; Marotta et al., 2019). However, in some clinical trials, lack of evidence of an effect on depression and related symptoms have also been reported either in depressed (Romijn et al., 2017) as well as in healthy individuals (in particular in older adults) (summarised in Butler et al., 2019) even though probiotic strains used were also previously successfully applied.

Probiotic supplements used in clinical trials for the treatment of depression, either alone (Akkasheh et al., 2016; Chahwan et al., 2019; Pinto-Sanchez et al., 2017), in combination with prebiotics (i.e. galactooligosaccharides) (Kazemi et al., 2019), or as adjunctive therapy with antidepressants (i.e. sertraline) (Eskandarzadeh et al., 2019) mainly include Lactobacillus (L.acidophilus, L. helveticus, L. brevis, L. casei, and L. salivarius), Lactococcus (L. lactis) and Bifidobacterium species (B. bifidum, B. lactis, and B. longum). Generally speaking, such treatments led to (1) a significant reduction of depression scores on Hospital Anxiety and Depression Scale and improvement of the cognitive reactivity scores in mild/moderate depression patients (Chahwan et al., 2019; Pinto-Sanchez et al., 2017), (2) a decrease of the anxiety symptoms in individuals with anxiety disorders (Eskandarzadeh et al., 2019) and (3) an improvement of the depression scores on Beck Depression Inventory in patients with a diagnosis of major depressive disorder (MDD) (Akkasheh et al., 2016; Kazemi et al., 2019).

In healthy subjects, administration of probiotics (L. casei Shirota, B. bifidum W23, B. lactis W52, L. acidophilus W37, L. brevis W63, L. casei W56, L. salivarius W24, and
L. lactis W19 and W58) improved the mood of subjects having lowest baseline mood levels and in general reduced the cognitive reactivity to sadness (Benton et al., 2007; Steenbergen et al., 2015).

Moreover, in other two studies led by Messaoudi et al. (2011) and Mohammadi et al. (2016), a significant reduction in overall anxiety and depression scores was shown after the treatment with L. helveticus R0052 and B. longum R0175 as well as with a polybiotic combination of various Lactobacillus strains (L. acidophilus, L. delbrueckii subsp. bulgaricus, L. casei, and L. rhamnosus), Bifidobacterium (B. breve, B. longum) and Streptococcus thermophilus strains. Besides mood, anxiety and depression scores, it has also been shown that the short-term administration of L. gasseri improved stress-associated symptoms in terms of sleep disturbance (Nishida et al., 2019).

Within the framework of the impact of probiotics on mood, we have previously reported (Marotta et al., 2019) on a double-blind, placebo-controlled study on 33 healthy volunteers who received daily either a probiotic mixture containing Limosilactobacillus fermentum LF16, Lacticaseibacillus rhamnosus LR06, Lactiplantibacillus plantarum LP01 (former members of Lactobacillus genus, Zheng et al., 2020), and B. longum 04 in maltodextrin, or a maltodextrin-only placebo, for 6 weeks, followed by a 3-weeks washout (Figure 1). Data obtained showed that the probiotics exerted a general improvement and persistence over time in different aspects of the mood state, including sadness, anger, and fatigue, accompanied by improvement in the sleep quality, which indicates that probiotics may increase the production of neuroactive precursors involved in emotional modulation, brain functions and circadian

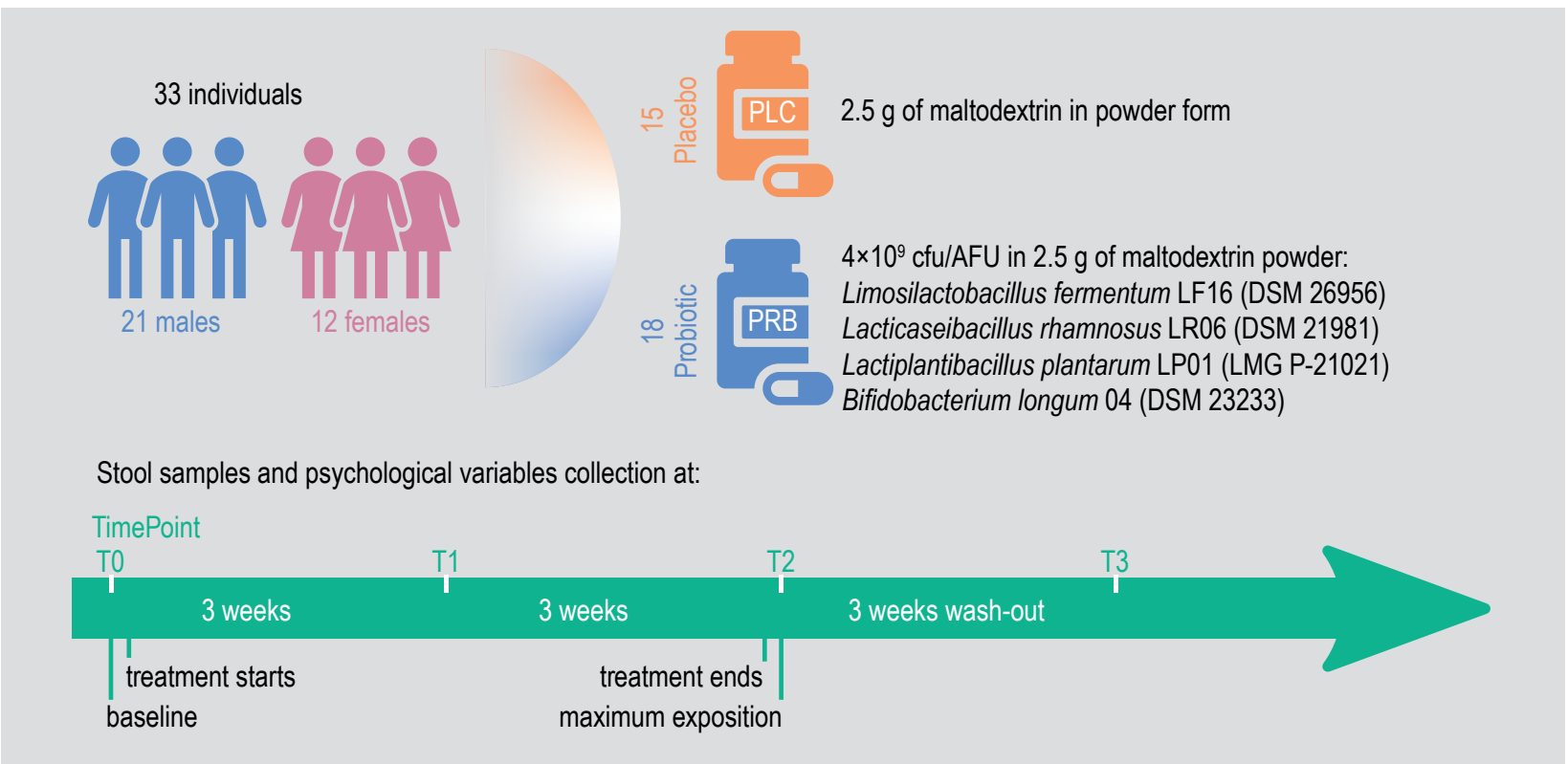

Figure 1. Experimental design of the study. 
rhythms. These findings corroborated the positive effect of probiotics on mental well-being, possibly determining changes in cognitive strategies to deal with problems by reducing sensitivity to negative situations.

The aim of the present study was to apply metabarcoding analysis of the faecal microbiota to determine (1) the microbial arrangement at baseline in the same cohort of healthy adults, which were randomised based on other characteristics, and (2) determine the effects of the probiotic and placebo consumption during and after the administration.

\section{Materials and methods}

\section{Sample collection}

The samples analysed derives from 33 healthy subjects enrolled in the study. Stool samples and psychological variables were collected at four time points (see Supplementary Table S1): before the intake of probiotic/ placebo (T0), at 3 (T1) and 6 (T2) weeks after the first intake and at the end of the third week of washout (T3).

The experimental group received 42 sachets of the product (one for each day), each containing $4 \times 10^{9} \mathrm{cfu} /$ active fluorescent units (AFU) of four probiotic species: L. fermentum LF16 (DSM 26956), L. rhamnosus LR06 (DSM 21981), L. plantarum LP01 (LMG P-21021), and B. longum 04 (DSM 23233) in $2.5 \mathrm{~g}$ of freeze-dried powder mixture containing maltodextrin (around $85 \%$ of the total weight) (Probiotical S.p.A., Novara, Italy). The control group received 42 sachets of placebo, each containing $2.5 \mathrm{~g}$ of maltodextrin in powder form. The placebo powder was indistinguishable from the probiotics powder in colour, taste, and smell. Participants were instructed to dissolve the powder in water or milk and drink it in the morning with breakfast. The probiotic sachets were analysed by Biolab Research S.r.l. (Novara, Italy), via flow cytometry (ISO 19344:2015 IDF 232:2015, $\geq 4 \times 10^{9}$ AFU) and plate count method (Biolab Research Method 014-06, $\geq 4 \times 10^{9} \mathrm{cfu}$ ) to confirm target cell count. Product stability was monitored to ensure minimum cell counts were maintained. The study was approved by the Ethical committee of Verona Hospital (Azienda Ospedaliera Universitaria Integrata, AOUI Verona, 766CESC) and it is registered in ClinicalTrials. gov with the number ID: NCT03539263.

\section{Library preparation and sequencing}

The 132 collected samples from 33 healthy subjects were stored at $-20{ }^{\circ} \mathrm{C}$ until analysis. DNA extraction and sequencing were performed at BMR Genomics S.r.l. (Padua, Italy). DNA was isolated with the Mobio Powerfecal kit (Mo Bio Laboratories, Inc., Carlsbad, CA, USA) adapted for QIAcube HT extractor (Qiagen, Hilden, Germany). V3-V4 regions of $16 \mathrm{~S}$ rRNA gene were amplified with previously described primers (Takahashi et al., 2014), modified with forward and reverse overhangs necessary for dual index library preparation (Illumina protocol (https://web.uri. edu/gsc/files/16s-metagenomic-library-prep-guide-

15044223-b.pdf) generating amplicons of $\sim 460 \mathrm{bp}$. The paired-end sequencing of the $16 \mathrm{~S}$ rRNA gene amplicons was performed using the MiSeq Illumina platform (dualindexing approach, $2 \times 300 \mathrm{bp}$ ) (Illumina, San Diego, CA, USA). A mock community was included as control. The resulting output was a set of 264 raw files in FASTQ format. All the reads have been submitted to SRA archive and are available under the bioproject PRJNA644097.

\section{Bioinformatics data analysis}

The whole analysis was performed on R (v3.6.1, R Core Team, 2019). Primarily, the FASTQ sequences were analysed using DADA2 (v1.13) (Callahan et al., 2016), a tool that implements an error correction model and allows to identify exact sample sequences that differ as little as a single nucleotide. The final output of DADA2 was an amplicon sequence variant (ASV) table which recorded the number of times each ASV was observed in each sample. DADA2 was run as described in https://benjjneb.github. io/dada2/bigdata.html using default parameters. In order to improve the overall quality of the sequences, the reads were filtered and trimmed using filterAndTrim function implemented in DADA2. Consequently, to remove low quality bases at the end of reads, the truncLen option was set to $(280,220)$ for the forward and reverse FASTQ files respectively. Similarly, to remove adapter sequences at the 5 ' end, the trimLeft option was set to $(17,21)$, for forward and reverse reads respectively. The removeBimeraDenovo function was used to remove chimeras, via consensus method, and then collapseNoMismatch function collapsed together all the reads that are identical up to shifts or length variation. Finally, the taxonomic assignment was performed using the naïve Bayesian classifier method implemented in DADA2 (assignTaxonomy and addSpecies functions) using as reference the EzBioCloud 16S database for QIIME pipeline (version 2018.05, https://www.ezbiocloud.net/ resources/16s_download), correctly formatted to work with the taxonomic classifier implemented within DADA2 (https://benjjneb.github.io/dada2/assign.html). A phylogenetic tree of the ASVs was obtained using the function AlignSeq implemented in DECIPHER (v2.12) (Wright, 2016), an R package to create multiple sequence alignments. FastTree (v2.1.10) (Price et al., 2010) was used to create the final tree.

\section{Data quality assessment and filtering}

Rarefaction curves on raw data were evaluated to assess the species richness among samples as a function of the sequencing depth. Data were pre-processed filtering taxa 
(ASVs) with low prevalence (where prevalence is the fraction of total samples in which an ASV is observed), setting a threshold of $0.5 \%$ for the cumulative relative abundance across all the samples; furthermore, taxa present in less than 2 samples were discarded. Synergistetes phylum members taxa (cumulative relative abundance $=0.34 \%$ ) and Lentisphaerae phylum members taxa (cumulative relative abundance $=0.03 \%$ ) were discarded by this filter. The pre-processing output data were then transformed to their relative abundances, and the 10 most present genera were plotted to phylum level. Mann-Whitney tests were performed on ASVs detected in these genera and the Benjamini \& Hochberg correction was applied to adjust the $\mathrm{p}$-values because of multiple testing.

In order to investigate the presence of probiotic related $\operatorname{tax} a$, a further taxonomy classification was performed. The softwares Kraken2 (Wood et al., 2019) and Bracken (Lu et al., 2017) were used to check both raw .fastq data and DADA2 inferred list of ASV, using two different pre-built Kraken2/Bracken databases (minikraken2_ v2_8GB_201904, k2_standard_16gb_20200919) and a custom database containing bacteria, archaea, virus, fungi and plants sequences, built using RefSeq (O'Leary et al., 2016) sequences.

\section{Biodiversity measurements}

Shannon-Wiener index was used to calculate $\alpha$-diversity, which was plotted stratifying the samples according to time points, gender and treatment type; the Kruskal-Wallis tests were performed to verify statistical differences in the $\alpha$-diversity among the samples. To measure $\beta$-diversity, data were normalised by three different methods (Cumulative Sum Scaling (CSS), Total Sum Scaling (TSS), Rarefaction) through the phyloseq_transform_css, phyloseq_standardize otu_abundance and rarefy_even_depth functions respectively. The first two functions are part of the vmikk/ metagMisc package (github.com/vmikk/metagMisc) while the latter belongs to the phyloseq package (v1.30.0) (McMurdie and Holmes, 2013). Each type of normalised data was inspected using four different distance metrics (Unweighted UniFrac, Weighted UniFrac, Bray-Curtis, Jaccard) and ordinated using the Principal Coordinates Analysis (PCoA) and Detrended Correspondence Analysis (DCA) ordination methods, through the ordinate function of the Vegan package (v2.5-5) (Oksanen et al., 2019).

A rigorous procedure was applied to evaluate the best combination of normalisation, distance metric, and ordination method. Normalization based on rarefaction was not considered as it performs very similarly to TSS due to the similar library sizes between samples.

At first, a hierarchical clustering was applied to the $\beta$-diversity bidimensional plot at the baseline grouping the samples in 3 and 4 groups. To test which of the two clustering methods performed better, the homogeneity of the cluster dispersions were tested using ANOVA F-test on betadisper function's output. A significant $P$-value indicated that the cluster dispersions were not homogeneous and that data needed to be taken with care. Secondly, the silhouette value was calculated, that is a measure of how similar an object is to its own cluster (cohesion) compared to other clusters (separation). The silhouette ranged from -1 to +1 , where a high value indicated that the object was well matched to its own cluster and poorly matched to neighbouring clusters. If most objects had a high value, then the clustering configuration was appropriate. On the other hand, if many samples had a low or negative value, then the clustering configuration might have too many or too few clusters. Finally, the cluster memberships found at the baseline were extended to all the other time points; cluster dispersions and silhouette indexes were computed again to verify the performances of the clustering on the whole dataset.

\section{Mixed effects regression models statistical analysis}

Amongst all the tested combinations, the TSS-normalised data, ordinated using the PCoA method and the unweighted-UniFrac distance metric, showed the most consistent results in cluster dispersions homogeneity and silhouettes, hence it was chosen for deeper exploration. To investigate the biological meaning of each PCoA coordinate, mixed-effects regression model analysis was performed on each, using the lme function of the nlme package (v3.1-140) (Pinheiro et al., 2020).

Firstly, the model formulation involved the Sample variable as a random component for each individual, and several categorical variables as fixed effects, such as TimePoint, Gender, Treatment and their interactions. Since all the variables were categorical, the regression framework set a baseline formed by TimePoint $=$ T0, Treatment $=$ Placebo and Gender = Female samples. Variable significance was guaranteed through an iterative process. Starting from the complete model, nonsignificant variables were dropped one by one. Every time a variable was dropped a log-likelihood ratio test (LRT) was performed in order to compare the likelihood of the model with the likelihood of the nested one $(P$-value $<0.1)$. This procedure allowed us to reach the most informative as well as parsimonious formulation of the model. Moreover, two versions of each model were compared: the first, where no correlation structure was specified, and the second, where the type of correlation was specified as an $\mathrm{AR}(1)$ process through the option correlation $=\operatorname{corAR} 1$ (form $=\sim 1 \mid$ Sample) of lme function.

Secondly, mixed-effects regression models were used to study correlation between sample variables and the PCoA components, with the new information about cluster 
memberships. The TimePoint, Gender, Treatment and Cluster variables were tested in the model, together with the interactions between TimePoint and Gender, TimePoint and Treatment, TimePoint and Cluster, Treatment and Cluster, and TimePoint, Treatment and Cluster. The already described model selection procedure was performed to choose the best model.

\section{Biomarkers investigation}

To retrieve information about the most discriminant features (Amplicon Sequence Variants, ASVs) of the clusters identified with the hierarchical clustering procedure, a discriminant analysis was computed using PLS-DA and sPLS-DA methods. Following the default mixOmics (v6.8) (Lê Cao et al., 2016; Rohart et al., 2017) pipeline (http:// mixomics.org/case-studies/splsda-srbct/), a pseudocount value of 1 was added to the counts table, which was then normalized with TSS and centered log-ratio (CLR) transformed. At first, the pipeline was performed on the clusters at baseline T0 to identify the most discriminant ASVs of each group. The discriminant analysis was then applied to each significant interaction resulted from the mixed-effects models, to investigate the effect of treatments. For each interaction a summary image was plotted using the HotLoadings function of the homonym package (github. com/mcalgaro93/HotLoadings), displaying the discriminant ASVs loadings and the related heatmap.

\section{Psychological variables analysis}

To find significant associations between psychological variables, treatments and clusters, Wilcoxon Rank Sum tests were performed between time points T0 and T1, T0 and $\mathrm{T} 2$, and $\mathrm{T} 0$ and $\mathrm{T} 3$ for placebo and probiotics groups. The $P$-values were also corrected for multiple testing using the Benjamini-Hochberg correction method.

\section{Results}

\section{$16 S$ metabarcoding sequencing depth and taxonomy classification}

A total of 5,382,700 paired-end sequences (an average of 40,778 reads per sample) with a read length of $300 \mathrm{bp}$ were obtained from the samples of the 33 subjects summarised in Figure 1. After read quality assessment, denoising and chimera filtering, 1,728 different ASVs were obtained. ASVs artefacts were removed with several filters and a total of 730 unique ASVs were obtained (Supplementary Figure S1). The taxonomy classification allowed to identify 10 phyla, 20 classes (730 ASVs), 27 orders (728 ASVs), 46 families (727 ASVs), 170 genera (720 ASVs) and 263 species (273 ASVs). The comparison of rarefaction curves (Supplementary Figure S2) as a function of sampling depth showed that all curves are close to saturation, therefore the richness of the samples has been fully observed or sequenced. The only exception was for subject number 8 at time point T2 that had a library size of 538 , while the second lower had a value of 8,848 ; for this reason, the former was discarded from the analysis.

The most abundant phylum was Firmicutes, with a relative frequency of $62.3 \%$ followed by Bacteroidetes 17.9\%, Proteobacteria 9.1\%, Verrucomicrobia 4.9\%, and Actinobacteria $4.7 \%$. The remaining $1 \%$ accounted for Euryarchaeota, Tenericutes, Saccharibacteria, Fusobacteria and Cyanobacteria. At genus level, the most abundant populations were Agathobacter, Blautia, Dialister, Faecalibacterium, Ruminococcus, Subdoligranulum (Firmicutes), Bacteroides (Bacteroidetes), Escherichia (Proteobacteria), Akkermansia (Verrucomicrobia), and Bifidobacterium (Actinobacteria) (Supplementary Figure S3).

\section{a-diversity analysis confirmed that the subjects of the cohort were comparable}

Samples were stratified according to TimePoint, Treatment (placebo or probiotics) and Gender using Shannon-Wiener index, as shown in Supplementary Figure S4. No significant differences among samples were observed, neither in the experimental nor in the control group (Kruskal-Wallis tests had $P>0.05)$. This finding was in line with expectations, as the subjects enrolled in the study were comparable when related to their internal diversity; neither alterations nor major shifts were expected on gut microbiota species richness or evenness regarding probiotic consumers.

\section{$\beta$-diversity analysis revealed three clusters and a strong sample-specific effect}

The flow chart in Supplementary Figure S5 summarizes the following steps of the analysis. All the $\beta$-diversity plots are shown in Supplementary Figure S6, while the homogeneity of cluster dispersions and silhouettes are presented in the Supplementary Results S1. The choice of the number of clusters was performed using only the samples at T0, which represents a snapshot of the microbiome composition before any type of treatment and allows to stratify the samples according to different bacteriotypes.

The identification of the best combination of number of clusters, normalisations, distances, and type of ordinations was then chosen. Specifically, the metrics that performed better in terms of homogeneity of cluster dispersions and silhouette values, when the cluster membership was extended also to all the other timepoints, were selected. Three clusters grouping with PCoA ordination method, based on unweighted UniFrac distances and TSS normalisation (Figure $2 \mathrm{~A}-\mathrm{C}$ ), produced the most consistent results (see Materials and methods 'Biodiversity 

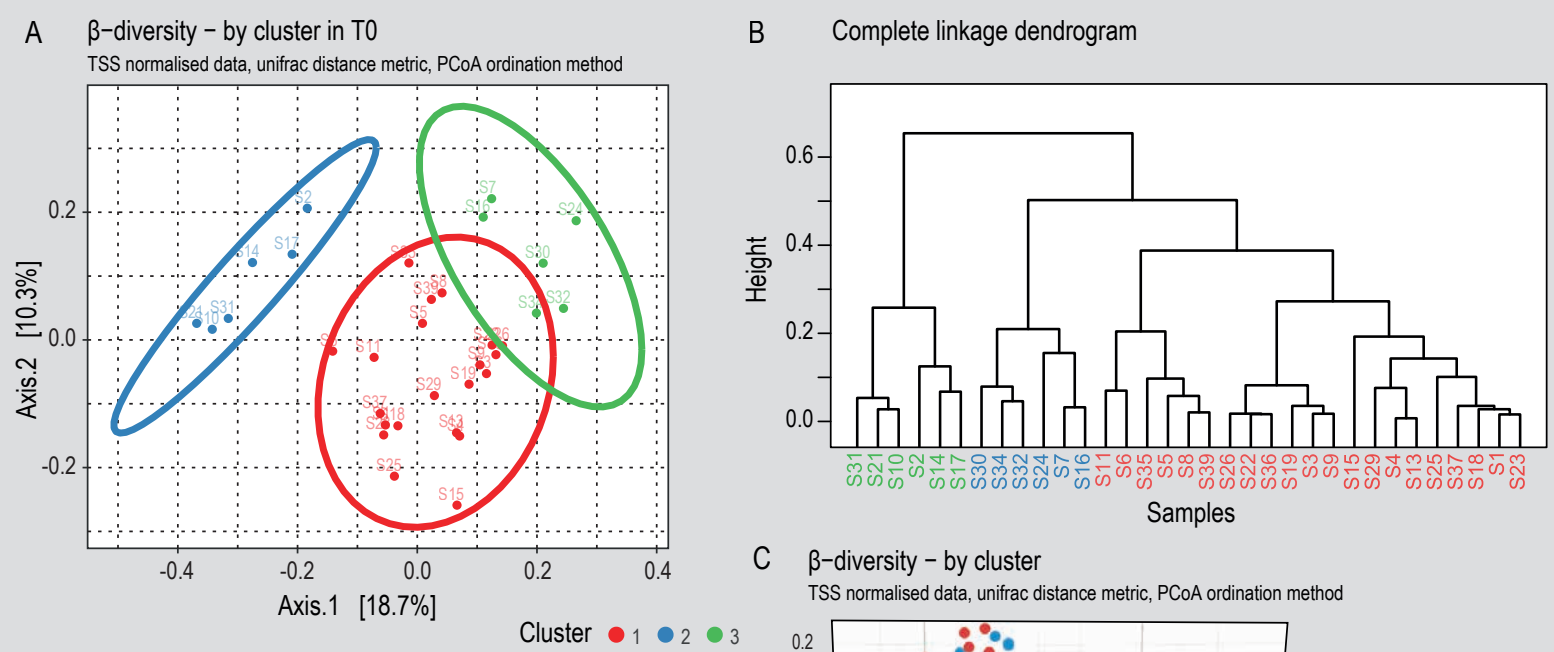

C $\beta$-diversity - by cluster

TSS normalised data, unifrac distance metric, $\mathrm{PCOA}$ ordination method

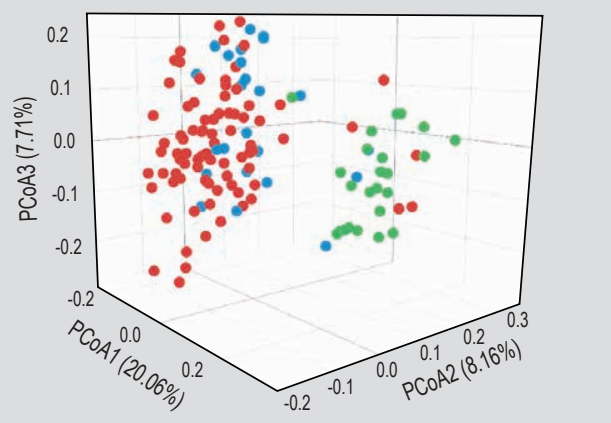

D

Mixed effects regression models Coefficients

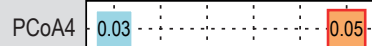

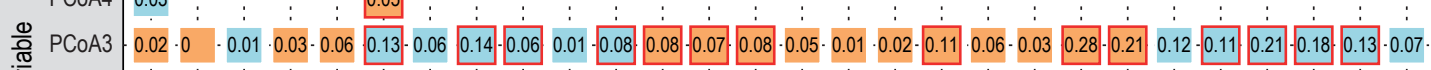

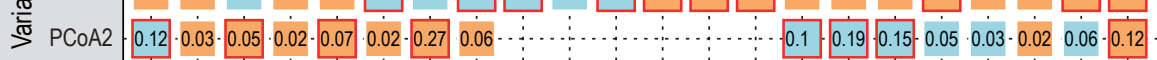

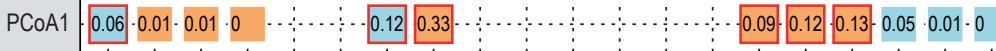
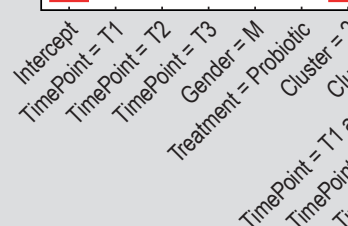

, $2^{2}, 3^{2}$

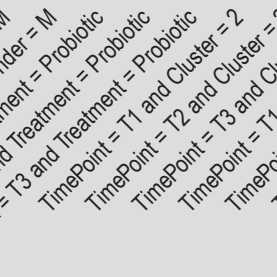

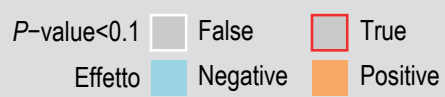

Model coefficient
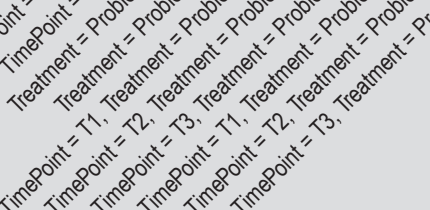

Figure 2. (A) Bidimensional representation of $\beta$-diversity for the samples in TimePoint $=$ TO (PCoA ordination method on UniFrac distance matrix of TSS normalized count data). Coloured by cluster membership obtained cutting the dendrogram in (B) in order to obtain 3 groups of individuals (see Methods for details of normalization, distance, and ordination's choice). (B) Hierarchical clustering dendrogram built with the complete linkage method on the euclidean distance matrix. Distance matrix based on PCOA1 and PCOA2 coordinates of the $\beta$-diversity for the samples in TimePoint $=$ TO (PCOA ordination method on UniFrac distance matrix of TSS normalised count data). (C) Tridimensional representation of $\beta$-diversity coloured by cluster membership for the samples in all time points (PCoA ordination method on UniFrac distance matrix of TSS normalised count data). (D) Linear mixed effects regression model coefficients. Blue (orange) coloured tiles represent a negative (positive) effect of the variable referred to the model baseline $($ TimePoint $=T 0$, Gender $=F$, Treatment $=$ Placebo, Cluster $=1)$. Statistically significant $(P<0.1)$ effects are represented by red squared tiles. 
measurements' and Supplementary Results S1 for details). Indeed, using these combinations of metrics, the stability of the clusters was maximised over time. In other words, the clusters identified at $\mathrm{T} 0$ tended to be the most consistent when the information of cluster membership is extended also to the other time points. The underlying idea was that the microbial signatures of the bacteriotype we identified at T0 should be stable over time, even though individual hosts may switch between enterotypes over long time periods (Moeller et al., 2012).

The composition of each cluster is reported in Table 1. As expected, samples of the same subject tended to form close subclusters, regardless the considered time point or treatment (Supplementary Figure S7). This suggests that the differences among subjects are stronger than the effects determined by the treatment.

\section{Mixed-effects regression models found associations between $\beta$-diversity and sample variables}

To inspect the variability held by the first four coordinates of the PCoA, four mixed-effect regression models were at first estimated without considering cluster membership (see Materials and methods). This regression framework allowed us to find significant correlations between PCoA coordinates and metadata such as Gender, Treatment and TimePoint and to remove sample-specific effects. In this context, we implicitly considered the Treatment=Placebo, Gender $=$ Female , and TimePoint $=T 0$ as the baseline level. As shown in Supplementary Figure S8, a correlation between the Treatment variable and the fourth component of the PCoA was found, while the third component showed a statistically significant difference in Gender at time point T3 compared to the baseline. This first analysis did not allow us to identify any statistically significant effects for the interactions between time points and treatments.

To further investigate if adding bacteryotype information would help in identifying significant effects for the interactions between time points and treatments, new mixed effects regression models were estimated adding the cluster membership variable as a fixed effect to the framework. Several significant interactions between time points and treatments were found for the third coordinate

Table 1. Cluster membership for individuals at the baseline $($ TimePoint $=$ T0).

$\begin{array}{lll}\text { Cluster } & \begin{array}{l}\text { Placebo treated } \\ \text { individuals }\end{array} & \begin{array}{l}\text { Probiotic treated } \\ \text { individuals }\end{array} \\ 1 & 9 & 12 \\ 2 & 3 & 3 \\ 3 & 3 & 3\end{array}$

(Figure 2D): (1) TimePoint $=$ T1, Treatment $=$ Probiotic and Cluster $=3$; (2) TimePoint $=$ T2, Treatment $=$ Probiotic and Cluster $=2$; (3) TimePoint $=$ T2, Treatment $=$ Probiotic and Cluster = 3; (4) TimePoint $=$ T3, Treatment $=$ Probiotic and Cluster $=2$. Clusters 2 and 3 were commonly affected by the variable TimePoint = T2 compared to the baseline: Cluster 2 responded later in the treatment (time points T2 and T3) while Cluster 3 responded at the beginning (time points T1 and T2). Although each interaction should be interpreted very carefully, these results highlighted a difference between the considered variable categories and the baseline (T0, Placebo, Cluster 1). Biologically speaking, the identified interactions could be an indicator of a distinct effect of the treatment considering different groups/bacteriotypes.

\section{sPLS-DA analysis showed that clusters were characterised by a specific bacteriotype}

A sPLS-DA analysis was performed to identify the most discriminant ASVs at the baseline T0. This multivariate approach identified two main components which were able to discriminate the clusters. The first component highlighted 5 taxa associated with Cluster 3 (Figure 3A,B): all the members of this group were characterised by the presence of SV33, assigned to Methanobrevibacter smithii, while $66 \%$ of them also displayed Sporobacter, Eubacterium and Oscillibacter spp. (SV168, SV256, SV37).

The second component highlighted the top 30 taxa associated with Clusters 1, 2 and 3 which created two different patterns as shown in the heatmap (Figure 3C,D). Cluster 1 individuals showed the general presence of Faecalibacterium spp. (SV3, SV14), while Cluster 3 were also characterised by Faecalibacterium spp. and Alistipes putredinis (SV4 and SV39); in Cluster 2, the second component revealed the presence of SV94-Eubacterium and SV77-Lachnospiraceae in almost all the members; 50\% of them were also characterised by Blautia spp. (SV562).

\section{Lacticaseibacillus rhamnosus is the only probiotic SV that increases significantly in the probiotic cohort in Cluster 1 and 2}

The sPLS-DA analysis revealed that SV232, associated with L. rhamnosus, was present in the probiotic cohort at time point T1 and T2 in Cluster 1 and Cluster 2, respectively (Supplementary Results S2 a, g). As for bifidobacteria, SV34 associated with B. longum was found to increase in Cluster 1 at T2 (where it was abundant also in the placebo individuals) and T3 Supplementary Results S2 e, k). Interestingly, other SVs associated with Bifidobacterium spp. displayed a different behaviour: SV228 was found to increase in the placebo cohort in Cluster 1 at T1 (Supplementary Results S2 b), while the relative abundance of SV121 and SV15 decreased in Cluster 1 and 2 at T3 (Supplementary Results $\mathrm{S} 2 \mathrm{l}, \mathrm{m})$. These observations showed that L. rhamnosus is 
A Loadings - First component sPLS-DA for samples in TimePoint $=$ T0

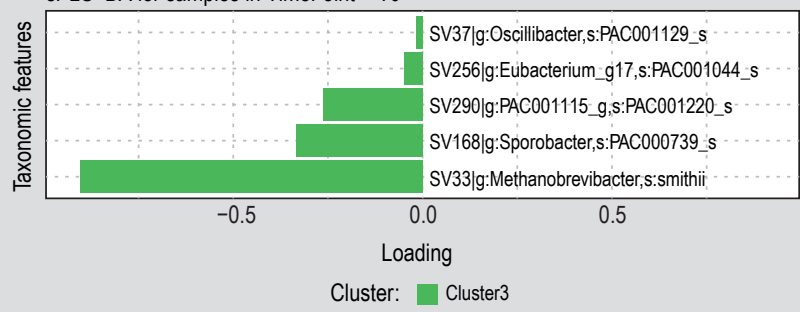

Loadings (top 40) - Second component

C sPLS-DA for samples in TimePoint = TO

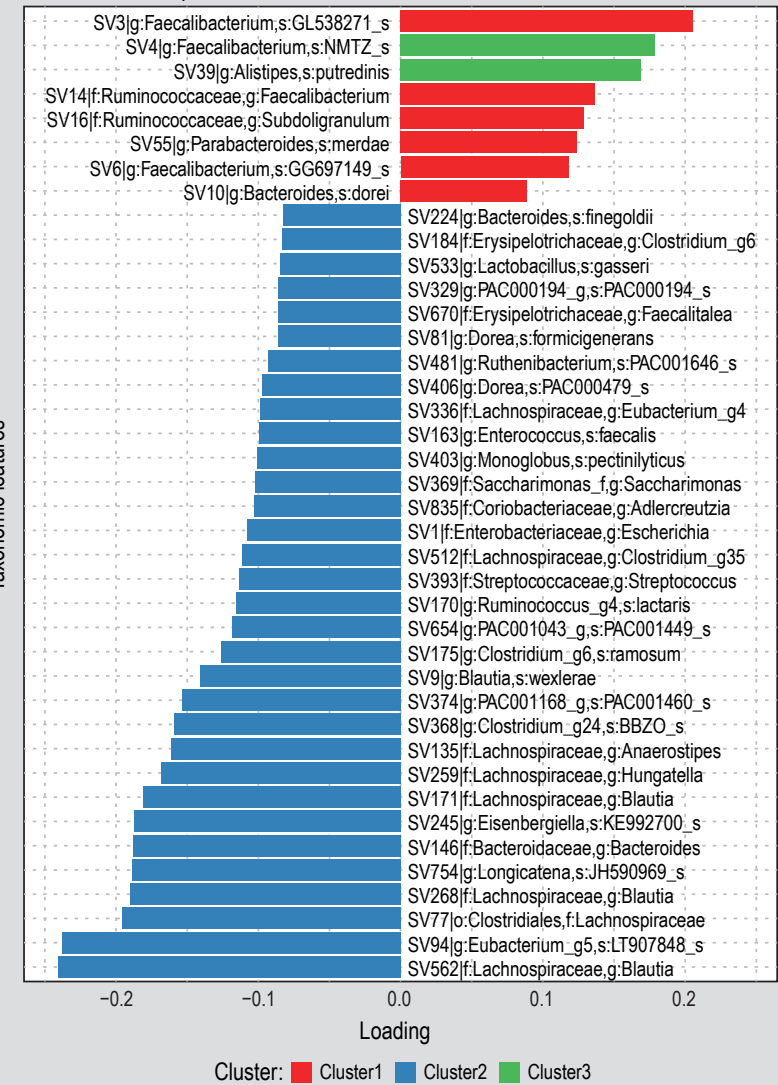

B Heatmap - First component

Samples in TimePoint = T0 - by Cluste

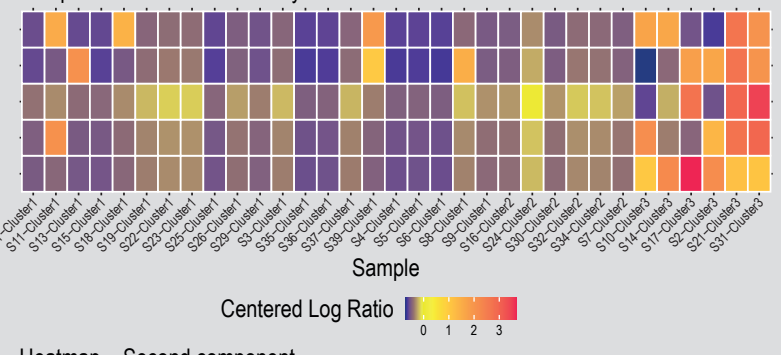

Heatmap - Second component

D Samples in TimePoint = T0 - by Cluster

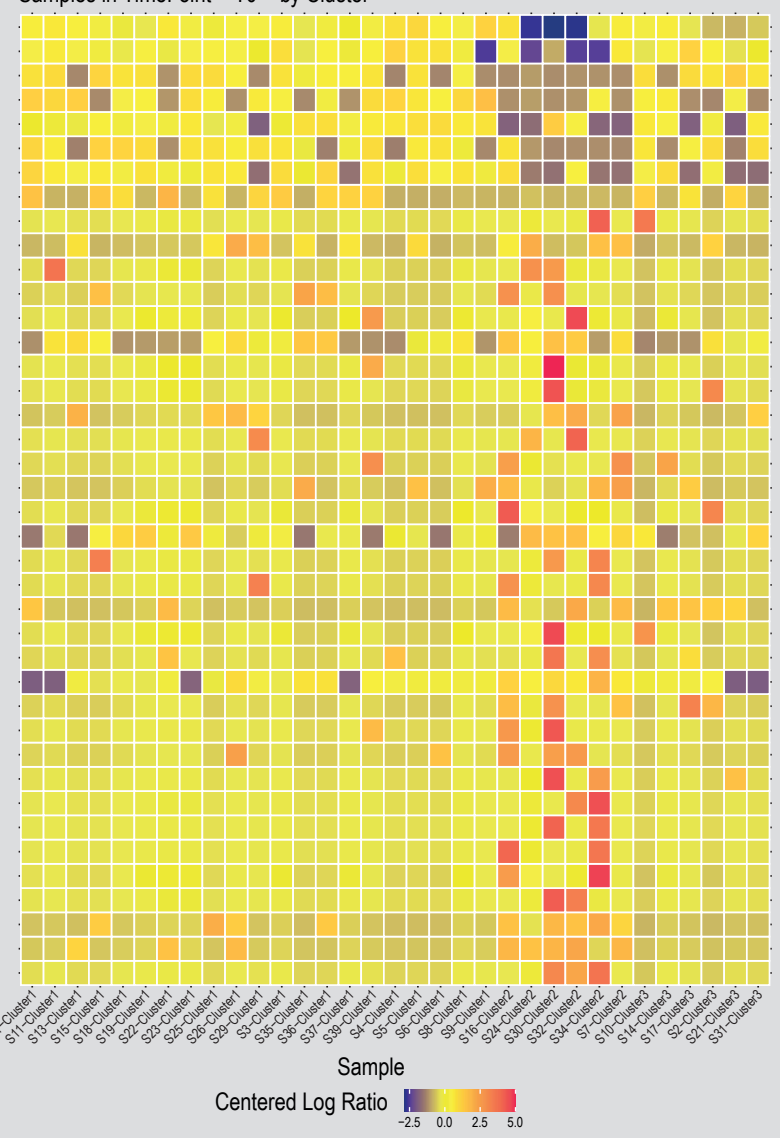

Figure 3. (A) SPLS-DA analysis at the baseline $($ TimePoint $=T 0)$. Loading values represent the 5 discriminant taxa of the first component, associated with Cluster 3 . Bigger the loading absolute value, stronger the association. (B) Heatmap shows the CLR values of the discriminant taxa in all the samples. (C) sPLS-DA analysis at the baseline (TimePoint $=$ TO). Loading values represent the first 30 (out of 135) most discriminant taxa of the second component, associated with Cluster 2 and Cluster 1. Bigger the loading absolute value, stronger the association. (D) Heatmap shows the CLR values of the discriminant taxa in all the samples.

the only probiotic SV that increases during the probiotic administration until T1 and T2 in Cluster 1 and Cluster 2 , respectively.

\section{Bacteriotypes changed distinctly in the probiotic and placebo cohorts}

The probiotic intake in Cluster 1 was associated with an increase of Coproiciproducens leptum (Clostridium letpum), Romboutsia timonensis and Mogibacterium spp. (SV264,
SV25 and SV664) from T1 to T3, respectively; the same cohort displayed a decrease of SVs related to Butyricimonas (SV785), Lachnospira (SV144) and Faecalibacterium spp. (SV4) at the same time points (Supplementary Results $\mathrm{S} 2 \mathrm{a}, \mathrm{e}, \mathrm{k})$. The placebo individuals featured a decrease of Butyricimonas, Alistipes, and Ruthenibacterium lactatiformans (SV774, SV465 and SV90) and a higher abundance of Anaerotignum (SV370), S. thermophilus (ST32) and Turicibacter spp. (SV56) from T1 to T3 (Supplementary Results S2 b, f, l). 
Individuals who took probiotics in Cluster 2 showed a significant decrease of Ruminococcaceae (SV348) in T2 (Supplementary Results S2 g) while the placebo group were characterised by an increment of Alistipes onderdonkii (SV73) and Lachnospiraceae spp. (SV144) in T2 and T3, respectively, and a drop of Blautia spp. (SV562) and Clostridium spp. (SV512) in the same time points (Supplementary Results S2 h, n).

In Cluster 3, Phascolarctobacterium faecium (SV97) and Subdoligranulum spp. (SV16) distinguished the probiotic cohort at T1 and T2 which, conversely, showed negative CLR values for Dialister invisus (SV5) and Eubacterium spp. (SV256) at the same time points (Supplementary Results S2 d, j); this latter species (SV94) increased together with Roseburia hominis (SV161) in the placebo subjects, which also showed a decrease of Intestinibacter bartlettii (SV63) and Bacteroides (SV47) at T1 and T2, respectively (Supplementary Results S2 c, i).

\section{Maltodextrin exerted an effect on the bacteriotype of each cluster}

Since maltodextrins are included both in the placebo and in the probiotic products, their impact on each cluster's bacteriotype (included Cluster 1) was investigated (Supplementary Results S3). Focusing on SVs related to probiotics, SV34-B. longum generally increased in members of Cluster 1 at T2 and T3 and in Cluster 2 at T1 (Supplementary Results S3 b, d, g); as for SVs related to other Bifidobacterium spp., a general reduction of SV121 and SV15 was observed in both probiotic and placebo groups in Cluster 1 at T2 and in Cluster 2 at T3 (Supplementary Results S3 g, h).

Considering other taxa, Cluster 1 was characterised by a general increase in relative abundance of $S$. thermophilus (SV32), R. timonensis (SV25), Turicibacter spp. (SV56), and a decrease of Butyricimonas spp. (SV774) and Lachnospira spp. (SV144) from T1 to T3 (Supplementary Results S3 a, d, g).

Cluster 2 individuals were characterised by higher levels of Faecalibacterium (SV14), and Roseburia inulinivorans (SV54) at T1 and T3; while SVs related to Escherichia (SV1), Agathobaculum (SV87), Blautia (SV17), and Eubacterium (SV94) decreased from T1 to T3 (Supplementary Results S3 b, e, h).

Finally, in Cluster 3 positive CRL values were associated to Anaerotignum, Pseudoflavonifractor and Sporobacter (SV282, SV428, SV145) while negative values were related to D. invisus (SV5), Bacteroides (SV140) and Blautia obeum (SV29) (Supplementary Results S3 c, f, i).

\section{Sequence variants related to Limosilactobacillus fermentum were detected in only one individual treated with probiotics}

ASVs associated with L. fermentum and L. plantarum, included in the probiotic product, were investigated and checked through the 16S-based ID tool of EzBioCloud. net, https://www.ezbiocloud.net/identify; database version 2020.10.12). SV1273 associated with L. fermentum was detected only in one probiotic cohort sample at T1, while conflicting results were obtained using different databases related to L. plantarum, confirming that the V3-V4 region for this species is not informative (Figure 4).

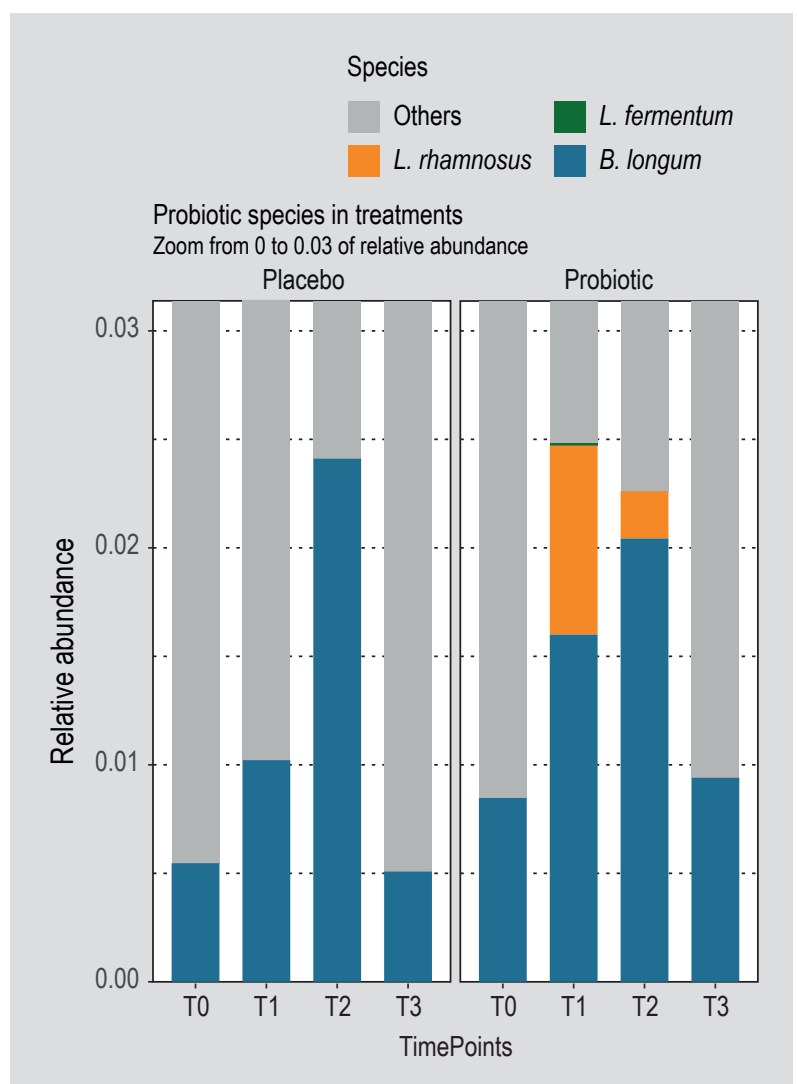

Figure 4. The species related to the probiotic compound were isolated and plotted in this barplot. The relative abundances percentages were zoomed to visualize the portion from 0 to 0.3 and stratified by time points and treatment type. The Bifidobacterium longum species is present in each time point for both the treatment types, showing a shared increasing trend. Limosilactobacillus fermentum was detected only for the second time point (T1) in the probiotic cohort. Lacticaseibacillus rhamnosus taxa were detected for both the second and the third time points, relative to the probiotic cohort. 


\section{Sleep quality and mood changes were detected in probiotics treated individuals of Cluster 1}

As shown in Figure 5A, a significant reduction $(P=0.03)$ was detected between time points T0-T1 and confirmed for T0-T2 and T0-T3 for the Pittsburgh Sleep Quality Index (PSQI). The PSQI global score is inversely correlated to the sleep quality (the lower the score, the better the sleep quality). The identified reduction indicates a sleep quality improvement for the probiotics treated individuals of Cluster 1.
Other significant changes were detected for the depression, anger, and fatigue subscales of the Profile and Mood State (POMS) psychological variables. Specifically, between T0-T1 and T0-T3 for anger $(P=0.08,0.02$; Figure $5 \mathrm{~B})$ and depression $(P=0.08,0.06$; Figure $5 C)$ indexes, and between T0-T1 $(P=0.04)$, T0-T2 $(P=0.02)$, and T0-T3 $(P=0.03)$ for the fatigue subscale (Figure 5D). It is noteworthy a clear descending trend for all mentioned psychological variables also in Cluster 3, even though these differences were not significant, probably due to the low sample size of the cluster. A similar pattern was not visible in Cluster 2.

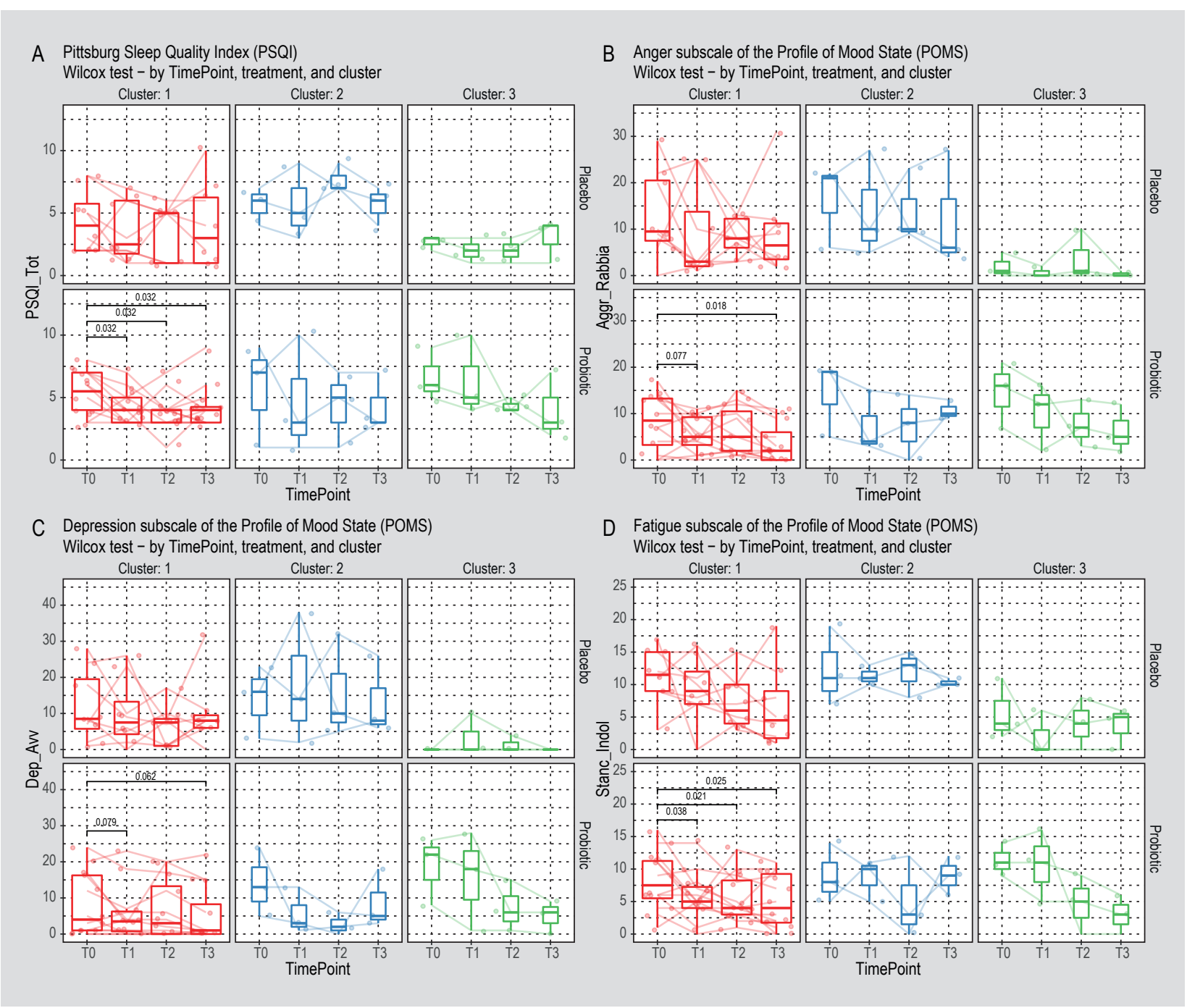

Figure 5. Wilcoxon Rank Sum tests between time points T0-T1, T0-T2, T0-T3. P-values are corrected for multiple testing using the Benjamini-Hochberg correction method and only adjusted $P$-values lower than 0.1 are reported. (A) Pittsburgh Sleep Quality Index (PSQI), stratified by cluster and treatment. (B) Anger subscale for the Profile of Mood State (POMS) psychological variable, stratified by cluster and treatment. (C) Depression subscale for the Profile of Mood State (POMS) psychological variable, stratified by cluster and treatment. (D) Fatigue subscale for the Profile of Mood State (POMS) psychological variable, stratified by cluster and treatment. 


\section{Discussion}

\section{Biodiversity measures stratified individuals in three clusters related to their microbiota}

In the present work the possible effect caused by the intake of B. longum, L. fermentum, L. rhamnosus and L. plantarum strains for 6 weeks (followed by a 3 week-washout) on the gut microbiota composition of a cohort of 33 healthy subjects was investigated.

A robust bioinformatic pipeline was implemented to analyse and characterise the metabarcoding data; a series of exploratory analyses were performed targeting particular effects with a possible biological correspondence, which could be related to the cognitive and emotional improvements we assessed in our previous study (Marotta et al., 2019). Biodiversity measures did not detect a significant diversity within the samples ( $\alpha$-diversity) but a samplespecific effect was found between samples ( $\beta$-diversity). This finding led to perform a statistical analysis using a mixedeffects model, through which several minor significant effects were found. The 33 individuals were clustered into three groups/bacteriotypes at the baseline; each one of them responded distinctly to the treatments. Cluster 1 and 2 were most impacted by the probiotic treatment, while Cluster 3 responded more to the placebo treatment. Furthermore, Cluster 1 responded throughout the whole treatment, while Cluster 2 and Cluster 3 had, respectively, a late and an early response. Those behaviours reinforce the concept that individuals with diverse bacteriotypes might respond differently to the same treatment. In addition, stratification of individuals according to their bacterial composition may be useful to better understand and predict the responses to specific treatments, such as probiotic interventions (Cheng and Ning, 2019; Christensen et al., 2018).

\section{Maltodextrin has a bifidogenic effect}

Focusing on SVs related to the probiotic species, it was observed that some Bifidobacterium-related SVs (SV15 and SV121) decreased both in the placebo and probiotic groups, but others, such as SV34-B. longum and SV228Bifidobacterium significantly increased in the placebo cohort both in Cluster 1 and Cluster 2. Their presence in the control subjects, who were administered maltodextrin, is also in line with data reported in a previous work (Watson et al., 2013) where authors observed that the majority of the culturable bifidobacterial strains (including 10 strains of $B$. longum) were capable of growing in maltodextrin rich media. Tandon and colleagues (2019) observed the same behaviour of the bifidobacterial population in a randomized, double-blind, placebo-controlled, doseresponse relationship study led to investigate the efficacy of fructo-oligosaccharides on human gut microbiota, where maltodextrin was used as control.
From this perspective, this study does not include a true placebo cohort which may have prevented to capture time dependent oscillations in abundances of relevant taxa; further, since maltodextrins are broadly used as placebo treatment, their bifidogenic effect needs to be deeply evaluated in future clinical trials involving bifidobacteria. Considering our data, we suggest that participants of this study were subjected to two different treatments rather than one: a 'synbiotic' administration (probiotics and maltodextrin) and a 'prebiotic' assumption (maltodextrin).

\section{Cluster 1 individuals displayed different gut composition following the prebiotic and synbiotic treatments}

The gut composition of individuals who received the synbiotics in Cluster 1 are selectively characterised by the presence of L. rhamnosus and C. leptum at the beginning of the treatments and then of Mogibacterium (described in 2000 to include strains isolated from the human periodontal environment; Nakazawa et al., 2000) at the end. C. leptum belongs to Clostridium Cluster IV which was reduced in patients with depression and anhedonia and it was negatively associated with scores in Quick Inventory of Depressive Symptoms-Self-Rated (QIDS-SR) and the Generalized Anxiety Disorder (GAD)-7 (Mason et al., 2020). The effect of probiotics administration on the abundance of C. leptum was also observed by Sato and colleagues (2017) where patients with type-2 diabetes had higher counts of C. leptum after 16 weeks of probiotics (L. casei Shirota) assumption. The synbiotic treatment specifically reduced the levels of Faecalibacterium, which was among the signature taxa of this cluster. This taxon is usually lower in MDD patients but there is still a lack of congruence across investigations (Barandouzi et al., 2020; Cheung et al., 2019).

The prebiotic treatment is associated with higher levels of Anaerotignum (described in 2017 following the isolation of strains from a methanogenic reactor; Ueki et al., 2017), S. thermophilus, Turicibacter and D. invisus. Among these species, it is interesting to report a reduced abundance of Turicibacter spp. was observed in socially defeated mice and was strongly correlated to pro-inflammatory cytokine changes within the prefrontal cortex (Szyszkowicz et al., 2017). However, this has to be further investigated, as Turicibacter levels were also found to be higher in depressed subjects (Kelly et al., 2016). As for D. invisus, it is usually lower in MDD patients and in other autoimmune diseases, including Crohn's disease, ulcerative colitis and rheumatoid arthritis (Lee et al., 2019).

Prebiotic administration was also related to reduced levels of Butyricimonas, Lachnospira, Alistipes and R. lactatiformans (isolated in 2016 from human faeces; Shkoporov et al., 2016). The decrease of Butyricimonas spp. may have a positive effect on the individuals: Butyricimonas members 
were found to be at higher levels in the gut microbiota of patients with clinically significant depression compared to control patients (Jiang et al., 2015). As for Lachnospira, no consensus data have been obtained so far, as Cheung and colleagues reported that this taxon could be related to MDD as well as to healthy subjects (Barandouzi et al., 2020; Cheung et al., 2019).

A significant association with depression was shown for Alistipes both in human cases as well as in mice subjected to stress over an extended time period. Since high levels of this taxon in the gut microbiota were also linked to chronic fatigue syndrome and irritable bowel syndrome (IBS), it has been suggested that Alistipes may promote depression through inflammatory pathways. In addition, Alistipes species are indole-positive and may thus influence tryptophan availability (the precursor of serotonin), disrupting the balance in the intestinal serotonergic system (Naseribafrouei et al., 2014). They are also high metabolisers of proteins and amino acids and, as such, they could trigger the production of toxic compounds such as ammonia, putrescine, and phenol (Cheung et al., 2019). However, these data are not concordant with what observed from Zheng and colleagues who reported that Alistipes were overrepresented in healthy control subjects compared to patients diagnosed with MDD (Zheng et al., 2016).

\section{Prebiotic treatment in Cluster 2 has an opposite effect of the synbiotic in Cluster 1}

In Cluster 2, the prebiotic supplementation induced a general oscillation of the abundance levels of Eubacterium, Blautia and Lachnospiraceae spp., which characterized the bacteriotype of this group. Kim and colleagues (2020) suggested that the reduction in the relative abundances of Eubacterium is related to the increase of the brainderived neurotrophic factor in the serum, improving brain functions. On the contrary respect to Cluster 1, the comparison between the prebiotic and synbiotic treatments showed that Alistipes spp. and Lachnospira spp. increased in Cluster 2 individuals.

The synbiotic intervention specifically led to a lower abundance of Ruminococcaceae (heterotypic synonym of family Oscillispiraceae): at family level, it was observed that these taxa were lower in depressed subjects compared to the control group (Jiang et al., 2015) and were correlated with behavioural changes induced by stress in mice (Bangsgaard Bendtsen et al., 2012). Conversely, prebiotics reduced the levels of Blautia, Clostridium, Escherichia and Agathobaculum spp.: although no data have been reported yet on the association of Agathobaculum (a strictly anaerobic and butyrate-producing strain isolated from the faeces of a healthy 23-year-old Korean female, Ahn et al., 2016), with stress-related disorders and there is a lack of consensus related to the presence of Escherichia in MDD patients, this effect could be considered beneficial for this cluster, as both Blautia spp. and Clostridium are usually found at higher levels in patients with MDD (Cheung et al., 2019; Jiang et al., 2015). Conversely, Faecalibacterium and $R$. inulinivorans increased at the end of prebiotic treatment: this species has been shown to have beneficial effects in specific conditions (i.e. atherosclerosis, Liu et al., 2020a) but no particular correlation has been found with mental or stress-related disorders (Cheung et al., 2019).

\section{Treatments in Cluster 3 changed the relative abundance of Phascholarctobacterium faecium, Subdoligranulum and Eubacterium spp.}

The assumption of the synbiotic in Cluster 3 individuals increased the relative abundance of Subdoligranulum and P. faecium and Eubacterium spp. (which characterised the bacteriotype at the baseline). It is interesting to note that P. faecium and, in general, family Acidaminococcaceae are more correlated to patients with active MDD (Jiang et al., 2015) and with both IBS and depression (Jeffery et al., 2012) rather that with healthy subjects. On the contrary, Subdoligranulum are depleted in subjects with IBS and depression, so its presence in the synbiotic cohort can be interpreted as a positive effect of this treatment (Liu et al., 2020b). This taxon is capable of producing short chain fatty acids (in particular butyrate) that protect the intestinal mucosa and regulate the immune system. More specifically, short-chain fatty acids (SCFA) play an important role in the differentiation of $\mathrm{T}$ cells and as histone deacetylase inhibitors, which were found to have immunosuppressive and anti-inflammatory functions and have been explored as potential novel antidepressants (Cheung et al., 2019). $R$. hominis, Anaerotignum spp. (similarly to Cluster 1), Pseudoflavonifractor, Eubacterium (conversely to the synbiotic treatment) and Sporobacter (among the signature taxa of this Cluster) were significantly abundant following the prebiotic treatment while I. bartletti, Bacteroides and B. obeum decreased. Sporobacter and Pseudoflavonifractor are among the common taxa found in the human gut microbiota; as for $R$. hominis, although no data are available regarding the direct positive or negative connection of this species and stress-related disorders, the reduction in the abundance of butyrate-producing Lachnospiraceae members, (including $R$. hominis) which are beneficial for the integrity and function of intestinal barrier, was involved in the formation of stress-induced visceral hypersensitivity for which $R$. hominis was proposed as a candidate potential probiotic (Zhang et al., 2019). As for I. bartlettii, it is interesting to report that it was found more frequently in the faecal samples of children with neurodevelopmental disorders compared to the control subjects (Bojovic et al., 2020). Finally, Bacteroides spp. exhibited divergent directionality and were found to be associated both with MDD as well as with healthy status, so no conclusions 
can be made on the effect of their reduction in this cohort (Barandouzi et al., 2020).

\section{Conclusions}

Although no consensus observations on the biological significance of particular components of the gut microbiota on mood disorders have been obtained yet, the present study shows that both the 'synbiotic' and the 'prebiotic' intake over a period of 6 weeks significantly changed the composition of the gut microbiota.

A debate is still ongoing whether the probiotic supplementation alters successfully the microbiota composition (Kristensen et al., 2016); in this perspective, Pinto-Sanchez and colleagues (2017) observed that probiotic administration in patients with IBS led to changes in urine metabolic profiles, brain activity and to antidepressant effects, but no detectable effects on the gut microbiota composition were noticed. Nevertheless, it has been demonstrated that probiotic treatments impact on both the gut microbiota gene expression (with potential antiinflammatory effects) and the gut barrier function (as also shown by the probiotic strains used in the present study - Pane M, personal communication); which can lead to an effect on the cognitive function (Chahwan et al., 2019).

Overall, this study offers evidence that probiotics supplementation has variable impacts depending on the gut microbiota bacteriotypes (i.e. Cluster 1, 2 and 3). In some cases, the shifts were towards microbial populations generally related to a healthy mood status (i.e. higher abundance of C. leptum and Subdoligranulum in Cluster 1 and 3, respectively) and suggests some mechanisms (i.e. SCFA production related to Subdoligranulum) which might rationalise the positive effects of the supplementation on the depressive mood state and sleep quality we observed in our previous work (Marotta et al., 2019). Particularly, variations of microbiota compositions were found to be statistically related to sleep quality improvement and to a descending rate of depression, anger and fatigue in probiotic-treated individuals of Cluster 1 .

Overall, these findings should be interpreted with caution: first of all, further studies are necessary on a larger and more homogeneous cohort of individuals, taking fully into account the effects of gender, diet, body mass index, presence of inflammation, bacteriotypes, and other factors that may be important covariates affecting the faecal microbiota.

As for diet, it is well established that it is one of the major modulators of the microbiota, therefore its monitoring is of utmost interest to better understand microbial dynamics and link them to other metabolic and physiological parameters (Bowyer et al., 2018). However, the monitoring of young healthy individuals for 6 weeks (9 including washout) proved to be a very challenging task, with too partial data that could not be used for associations.

Indeed, in this study, we tried to move from an effectiveness perspective (the whole cohort) to an efficacy-focused one (the clusters/bacteriotypes), revealing some complexities on the microbial background related to the effects described by Marotta et al. (2019) on almost the same cohort. Shedding light on these variables, especially on a healthy individual's cohort, is expected to allow a better development of psychobiotic treatment strategies. This will contribute to the definition of probiotics as an adjunct therapy or for the prevention of mood-related disorders.

\section{Supplementary material}

Supplementary material can be found online at https://doi. org/10.3920/BM2020.0137.

Table S1. General information about samples and psychological variables.

Figure S1. Taxa prevalence exploration and prevalence filtered taxa.

Figure S2. Rarefaction curves as a function of sampling depth.

Figure S3. The top 10 genera and related phyla of membership are depicted in this image; taxa are plotted for their relative abundance over the different time points, facetted for the different type of treatment.

Figure S4. Shannon-Wiener $\alpha$-diversity, over time points, treatment type and faceted by gender.

Figure S5. Statistical analysis steps from $\beta$-diversity ordination choice, clustering, and the mixed-effects regression models.

Figure S6. Bidimensional representation of $\beta$-diversities.

Figure S7. Bidimensional representation of $\beta$-diversity by timepoint and treatment, and by sample.

Figure S8. Linear mixed effects regression model coefficients.

\section{Supplementary Results S1-S3.}

\section{Acknowledgements}

Nina Vinot contributed to the scientific linguistic revision of the manuscript. 
This project was also funded by Cibo intelligente per un future sostenibile - 3S_4H - Safe, Smart, Sustainable food for Health (Domanda di sostegno ID 10065201) FESR Regione Veneto Bando DGR n. 1139 del 19/07/2017 Asse 1. Azione 1.1.4 'Bando per il sostegno a progetti di Ricerca e Sviluppo sviluppati dai Distretti Industriali e dalle Reti Innovative Regionali' - POR FESR 2014-2020.

\section{Conflict of interests}

Marco Pane and Angela Amoruso are employed by Probiotical Research Srl (Novara, Italy). Microbion is a contract research organization providing support to clinical trials, but no conflict of interest is foreseen within the present study.

\section{References}

Ahn, S., Jin, T.E., Chang, D.H., Rhee, M.S., Kim, H.J., Lee, S.J., Park, D.S. and Kim, B.C., 2016. Agathobaculum butyriciproducens gen. nov. sp. nov., a strict anaerobic, butyrate-producing gut bacterium isolated from human faeces and reclassification of Eubacterium desmolans as Agathobaculum desmolans comb. nov. International Journal of Systematic and Evolutionary Microbiology 9: 3656-3661. https://doi.org/10.1099/ijsem.0.001195

Akkasheh, G., Kashani-Poor, Z., Tajabadi-Ebrahimi, M., Jafari, P., Akbari, H., Taghizadeh, M., Memarzadeh, M.R., Asemi Z. and Esmaillzadeh, A., 2016. Clinical and metabolic response to probiotic administration in patients with major depressive disorder: a randomized, double-blind, placebo-controlled trial. Nutrition. 32: 315-320. https://doi.org/10.1016/j.nut.2015.09.003

Bangsgaard Bendtsen, K.M., Krych, L., Sorensen, D.B., Pang, W., Nielsen, D.S., Josefsen, K., Hansen, L.H., Sorensen, S.J. and Hansen, A.K., 2012. Gut microbiota composition is correlated to grid floor induced stress and behavior in the BALB/c mouse. PLoS ONE 7: e46231. https://doi.org/10.1371/journal.pone.004623

Barandouzi, Z.A., Starkweather, A.R., Henderson, W.A., Gyamfi, A. and Cong, X.S. 2020. Altered composition of gut microbiota in depression: a systematic review. Frontiers in Psychiatry 11: 541. https://doi.org/10.3389/fpsyt.2020.00541

Bastiaanssen, T.F.S., Cussotto, S., Claesson, M.J., Clarke, G., Dinan, T.G. and Cryan, J.F., 2020. Gutted! Unraveling the role of the microbiome in major depressive disorder. Harvard Review of Psychiatry 28: 25639. https://doi.org/10.1097/HRP.0000000000000243

Benton, D., Williams, C. and Brown, A., 2007. Impact of consuming a milk drink containing a probiotic on mood and cognition. European Journal of Clinical Nutrition 61: 355-361. https://doi.org/10.1038/ sj.ejcn. 1602546

Bojović, K., Ignjatović, Đ.I., Soković Bajić, S., Vojnović Milutinović, D., Tomić, M., Golić, N. and Tolinački, M., 2020. Gut microbiota dysbiosis associated with altered production of short chain fatty acids in children with neurodevelopmental disorders. Frontiers in Cellular and Infection Microbiology 10: 223. https://doi.org/10.3389/ fcimb.2020.00223
Bowyer, R.C.E., Jackson, M.A., Pallister, T., Skinner, J., Spector, T.D., Welch, A.A. and Steves, C.J., 2018. Use of dietary indices to control for diet in human gut microbiota studies. Microbiome 6: 77. https:// doi.org/10.1186/s40168-018-0455-y

Butler, M.I., Sandhu, K., Cryan, J.F. and Dinan, T.G., 2019. From isoniazid to psychobiotics: the gut microbiome as a new antidepressant target. British Journal of Hospital Medicine 80: 139-145. https://doi.org/10.12968/hmed.2019.80.3.139

Callahan, B.J, McMurdie, P.J., Rosen, M.J., Han, A.W., Johnson, A.J.A. and Holmes, S.P., 2016. DADA2: high-resolution sample inference from Illumina amplicon data. Nature Methods 13: 581-583. https:// doi.org/10.1038/nmeth.3869

Chahwan, B., Kwan, S., Isik, A., Van Hemert, S., Burke, C. and Roberts, L., 2019. Gut feelings: a randomised, triple-blind, placebo-controlled trial of probiotics for depressive symptoms. Journal of Affective Disorders 253: 317-326. https://doi.org/10.1016/j.jad.2019.04.097

Cheng, M. and Ning, K., 2019. Stereotypes about enterotype: the old and new ideas. Genomics, Proteomics and Bioinformatics 17: 4-12. https://doi.org/10.1016/j.gpb.2018.02.004

Cheung, S.G., Goldenthal, A.R., Uhlemann, A.-C., Mann, J.J., Miller, J.M. and Sublette, M.E., 2019. Systematic review of gut microbiota and major depression. Frontiers in Psychiatry 10: 34. https://doi. org/10.3389/fpsyt.2019.00034

Christensen, L., Roager, H.M., Astrup, A. and Hjorth, M.F., 2018. Microbial enterotypes in personalized nutrition and obesity management. American Journal of Clinical Nutrition 108: 645651. https://doi.org/10.1093/ajcn/nqy175

Eskandarzadeh, S., Effatpanah, M., Khosravi-Darani, K., Askari, R., Hosseini, A.F., Mahnaz, R. and Jazayeri, S., 2019. Efficacy of a multispecies probiotic as adjunctive therapy in generalized anxiety disorder: a double blind, randomized, placebo-controlled trial. Nutritional Neuroscience 24: 102-108. https://doi.org/10.1080/1 028415X.2019.1598669

Jeffery, I.B., O’Toole, P.W., Öhman, L., Claesson, M.J., Deane, J., Quigley, E.M.M. and Simrén, M., 2012. An irritable bowel syndrome subtype defined by species-specific alterations in faecal microbiota. Gut 61: 997-1006. https://doi.org/10.1136/gutjnl-2011-301501

Jiang, H., Ling, Z., Zhang, Y., Mao, H., Ma, Z., Yin, Y., Wang, W., Tang, W., Tan, Z., Shi, J., Li, L. and Ruan, B., 2015. Altered fecal microbiota composition in patients with major depressive disorder. Brain, Behavior, and Immunity 48: 186-194. https://doi.org/10.1016/j. bbi.2015.03.016

Kazemi, A., Noorbala, A.A, Azam, K., Eskandari, M.H. and Djafarian, K., 2019. Effect of probiotic and prebiotic vs placebo on psychological outcomes in patients with major depressive disorder: a randomized clinical trial. Clinical Nutrition 38: 522-528. https:// doi.org/10.1016/j.clnu.2018.04.010

Kelly, J.R., Borre, Y., O' Brien, C., Patterson, E., El Aidy, S., Deane, J., Kennedy, P.J., Beers, S., Scott, K., Moloney, G., Hoban, A.E., Scott, L., Fitzgerald, P., Ross, P., Stanton, C., Clarke, G., Cryan, J.F. and Dinan, T.G., 2016. Transferring the blues: depression-associated gut microbiota induces neurobehavioural changes in the rat. Journal of Psychiatric Research 82: 109-118. https://doi.org/10.1016/j. jpsychires.2016.07.019 
Kelly, J.R., O’ Keane, V., Cryan, J.F., Clarke, G. and Dinan, T.G., 2019. Mood and microbes: gut to brain communication in depression. Gastroenterology Clinics of North America 48: 389-405. https:// doi.org/10.1016/j.gtc.2019.04.006

Kim C.-S., Lina, C., Sim, M., Jung, S., Chun, W.Y., Baik, H.W. and Shin, D.-M., 2020. Probiotic supplementation improves cognitive function and mood with changes in gut microbiota in communitydwelling elderly: a randomized, double-blind, placebo-controlled, multicenter trial. Journal of Gerontology Series A 76: 32-40. https:// doi.org/10.1093/gerona/glaa090

Kristensen, N.B, Bryrup, T., Allin, K.H, Nielsen, T., Hansen, T.H. and Pedersen, O., 2016. Alterations in fecal microbiota composition by probiotic supplementation in healthy adults: a systematic review of randomized controlled trials. Genome Medicine 8: 52. https:// doi.org/10.1186/s13073-016-0300-5

Lê Cao, K.A., Costello, M.-E., Lakis, V.A., Bartolo, F., Chua, X.-Y., Brazeilles, R. and Rondeau, P., 2016. MixMC: a multivariate statistical framework to gain insight into microbial communities. PLoS ONE 11: e0160169. https://doi.org/10.1371/journal.pone.0160169

Lee, J.Y., Mannaa, M., Kim, Y., Kim, J., Kim, G.T. and Seo, Y.S., 2019. Comparative analysis of fecal microbiota composition between rheumatoid arthritis and osteoarthritis patients. Genes 10: 748. https://doi.org/10.3390/genes10100748

Liu, S., Zhao, W., Liu, X. and Cheng, L., 2020a. Metagenomic analysis of the gut microbiome in atherosclerosis patients identify crosscohort microbial signatures and potential therapeutic target. FASEB Journal 34: 14166-14181. https://doi.org/10.1096/fj.202000622R

Liu, T., Gu, X., Li, L.X., Li, M., Li, B., Cui, X. and Zuo, X.L., 2020 b. Microbial and metabolomic profiles in correlation with depression and anxiety co-morbidities in diarrhoea-predominant IBS patients. BMC Microbiology 20: 168. https://doi.org/10.1186/s12866-02001841-4

Lu, J., Breitwieser, F.P., Thielen, P. and Salzberg, S.L., 2017. Bracken: estimating species abundance in metagenomics data. PeerJ Computer Science 3: e104. https://doi.org/10.7717/peerj-cs.104

Marotta, A., Sarno, E., Del Casale, A., Pane, M., Mogna, L., Amoruso, A., Felis, G.E. and Fiorio, M., 2019. Effects of probiotics on cognitive reactivity, mood, and sleep quality. Frontiers in Psychiatry 10: 164. https://doi.org/10.3389/fpsyt.2019.00164

Mason, B.L., Li, Q., Minhajuddin, A., Czysz, A.H., Coughlin, L.A., Hussain, S.K., Koh, A.Y. and Trivedi, M.H., 2020. Reduced antiinflammatory gut microbiota are associated with depression and anhedonia. Journal of Affective Disorders 266: 394-401. https:// doi.org/10.1016/j.jad.2020.01.137

McMurdie, P.J. and Holmes, S., 2013. Phyloseq: an R package for reproducible interactive analysis and graphics of microbiome census data. PLoS ONE 8: e61217. https://doi.org/10.1371/journal. pone.0061217

Messaoudi, M., Lalonde, R., Violle, N., Javelot, H., Desor, D., Nejdi, A., Bisson, J.-F., Rougeot, C., Pichelin, M., Cazaubiel, M. and Cazaubiel, J.-M. 2011. Assessment of psychotropic-like properties of a probiotic formulation (Lactobacillus helveticus R0052 and Bifidobacterium longum R0175) in rats and human subjects. British Journal of Nutrition 105: 755-764. https://doi.org/10.1017/S0007114510004319
Moeller, A.H., Degnan, P.H., Pusey, A.E., Wilson, M.L., Hahn, B.H. and Ochman, H., 2012. Chimpanzees and humans harbour compositionally similar gut enterotypes. Nature Communications 3: 1179. https://doi.org/10.1038/ncomms2159

Mohammadi, A.A., Jazayeri, S., Khosravi-Darani, K., Solati, Z., Mohammadpour, N., Asemi, Z., Adab, Z., Djalali, M., TehraniDoost, M., Hosseini, M. and Eghtesadi, S., 2016. The effects of probiotics on mental health and hypothalamic-pituitary-adrenal axis: a randomized, double-blind, placebo-controlled trial in petrochemical workers. Nutritional Neuroscience 19: 387-395. https://doi.org/10.1179/1476830515Y.0000000023

Nakazawa, F., Sato, M., Poco, S.E., Hashimura, T., Ikeda, T., Kalfas, S., Sundqvist, G. and Hoshino, E., 2000. Description of Mogibacterium pumilum gen. nov., sp. nov. and Mogibacterium vescum gen. nov., sp. nov., and reclassification of Eubacterium timidum (Holdeman et al. 1980) as Mogibacterium timidum gen. nov., comb. nov. International Journal of Systematic and Evolutionary Microbiology 2: 679-688. https://doi.org/10.1099/00207713-50-2-679

Naseribafrouei, A., Hestad, K., Avershina, E., Sekelja, M., Linløkken, A., Wilson, R. and Rudi, K., 2014. Correlation between the human fecal microbiota and depression. Neurogastroenterology and Motility 26: 1155-1162. https://doi.org/10.1111/nmo.12378

Nishida, K., Sawada, D., Kuwano, Y., Tanaka, H. and Rokutan, K., 2019. Health benefits of Lactobacillus gasseri CP2305 tablets in young adults exposed to chronic stress: a randomized, doubleblind, placebo-controlled study. Nutrients 11: 1859. https://doi. org/10.3390/nu11081859

Oksanen, F.J., Blanchet, G., Friendly, M., Kindt, R., Legendre, P., McGlinn, D., Minchin, P.R., O'Hara, R.B., Simpson, G.L., Solymos, P., Stevens, H.H.M, Szoecs, E. and Wagner, H., 2019. Vegan: community ecology package. R package. Available at: https://cran.r-project.org/ web/packages/vegan/index.html

O'Leary, N.A., Wright, M.W., Brister, J.R., Ciufo, S., Haddad, D., McVeigh, R., Rajput, B., Robbertse, B., Smith-White, B., AkoAdjei, D., Astashyn, A., Badretdin, A., Bao, Y., Blinkova, O., Brover, V., Chetvernin, V., Choi, J., Cox, E., Ermolaeva, O., Farrell, C.M., Goldfarb, T., Gupta, T., Haft, D., Hatcher, E., Hlavina, W., Joardar, V.S., Kodali, V.K., Li, W., Maglott, D., Masterson, P., McGarvey, K.M., Murphy, M.R., O’Neill, K., Pujar, S., Rangwala, S.H., Rausch, D., Riddick, L.D., Schoch, C., Shkeda, A., Storz, S.S., Sun, H., ThibaudNissen, F., Tolstoy, I., Tully, R.E., Vatsan, A.R., Wallin, C., Webb, D., Wu, W., Landrum, M.J., Kimchi, A., Tatusova, T., DiCuccio, M., Kitts, P., Murphy, T.D. and Pruitt, K.D., 2016. Reference sequence (RefSeq) database at NCBI: current status, taxonomic expansion, and functional annotation. Nucleic Acids Research 44: D733-745. https://doi.org/10.1093/nar/gkv1189

Pinheiro, J., Bates, D., DebRoy, S., Sarkar, D., R Core Team, 2020. nlme: linear and nonlinear mixed effects models. R package, Vienna, Austria. Available at: https://CRAN.R-project.org/package=nlme 
Pinto-Sanchez, M.I., Hall, G.B., Ghajar, K., Nardelli, A., Bolino, C., Lau, J.T., Martin, F.-P., Cominetti, O., Welsh, C., Rieder, A., Traynor, J., Gregory, C., De Palma, G., Pigrau, M., Ford, A.C., Macri, J., Berger, B., Bergonzelli, G., Surette, M.G., Collins, S.M., Moayyedi, P. and Bercik, P., 2017. Probiotic Bifidobacterium longum NCC3001 reduces depression scores and alters brain activity: a pilot study in patients with irritable bowel syndrome. Gastroenterology 153: 448-459. https://doi.org/10.1053/j.gastro.2017.05.003

Price, M.N, Dehal, P.S. and Arkin, A.P., 2010. FastTree 2 - approximately maximum-likelihood trees for large alignments. PLoS ONE 5: e9490. https://doi.org/10.1371/journal.pone.0009490

Rohart, F., Gautier, B., Singh, A. and Lê Cao, K.-A., 2017. mixOmics: an $R$ package for omics feature selection and multiple data integration. PLoS Computational Biology 13: e1005752. https://doi.org/10.1371/ journal.pcbi. 1005752

Romijn, A.R., Rucklidge, J.J., Kuijer, R.G. and Frampton, C., 2017. A double-blind, randomized, placebo-controlled trial of Lactobacillus helveticus and Bifidobacterium longum for the symptoms of depression. Australian and New Zealand Journal of Psychiatry 51: 810-821. https://doi.org/10.1177/0004867416686694.

Sato, J., Kanazawa, A., Azuma, K., Ikeda, F., Goto, H., Komiya, K., Kanno, R., Tamura, Y., Asahara, T., Takahashi, T., Nomoto, K., Yamashiro, Y. and Watada, H., 2017. Probiotic reduces bacterial translocation in type 2 diabetes mellitus: a randomised controlled study. Scientific Reports 1: 12115. https://doi.org/10.1038/s41598017-12535-9

Shkoporov, A.N., Chaplin, A.V., Shcherbakova, V.A., Suzina, N.E., Kafarskaia, L.I., Bozhenko, V.K. and Efimov, B.A., 2016. Ruthenibacterium lactatiformans gen. nov., sp. nov., an anaerobic, lactate-producing member of the family Ruminococcaceae isolated from human faeces. International Journal of Systematic and Evolutionary Microbiology 66: 3041-3049. https://doi.org/10.1099/ ijsem.0.001143

Steenbergen, L., Sellaro, R., Van Hemert, S., Bosch, J.A. and Colzato, L.S., 2015. A randomized controlled trial to test the effect of multispecies probiotics on cognitive reactivity to sad mood. Brain, Behaviour and Immunity 48: 258-264. https://doi.org/10.1016/j. bbi.2015.04.003

Szyszkowicz, J.K., Wong, A., Anisman, H., Merali, Z. and Audet, M.-C., 2017. Implications of the gut microbiota in vulnerability to the social avoidance effects of chronic social defeat in male mice. Brain, Behavior, and Immunity 66: 45-55. https://doi.org/10.1016/j. bbi.2017.06.009

Takahashi, S., Tomita, J., Nishioka, K., Hisada, T. and Nishijima, M., 2014. Development of a prokaryotic universal primer for simultaneous analysis of bacteria and archaea using next-generation sequencing. PLoS ONE 9: e105592. https://doi.org/10.1371/journal. pone.0105592
Tandon, D., Haque, M.M., Gote, M., Jain, M., Bhaduri, A., Dubey, A.K. and Mande, S.S., 2019. A prospective randomized, doubleblind, placebo-controlled, dose-response relationship study to investigate efficacy of fructo-oligosaccharides (FOS) on human gut microflora. Scientific Reports 9: 5473. https://doi.org/10.1038/ s41598-019-41837-3

Ueki, A., Goto, K., Ohtaki, Y., Kaku, N. and Ueki, K., 2017. Description of Anaerotignum aminivorans gen. nov., sp. nov., a strictly anaerobic, amino-acid-decomposing bacterium isolated from a methanogenic reactor, and reclassification of Clostridium propionicum, Clostridium neopropionicum and Clostridium lactatifermentans as species of the genus Anaerotignum. International Journal of Systematic and Evolutionary Microbiology 10: 4146-4153. https://doi.org/10.1099/ ijsem.0.002268

Watson, D., O'Connell Motherway, M., Schoterman, M.H.C., Joost van Neerven, R.J., Nauta, A. and Van Sinderen, D. 2013. Selective carbohydrate utilization by lactobacilli and bifidobacterial. Journal of Applied Microbiology 114: 1132-1146. https://doi.org/10.1111/ jam.12105

Wood, D.E., Lu, J. and Langmead, B., 2019. Improved metagenomic analysis with Kraken 2. Genome Biology 20: 257. https://doi. org/10.1186/s13059-019-1891-0

Wright, E.S., 2016. Using DECIPHER v2.0 to analyze big biological sequence data in R. The R Journal 8: 352-359.

Zhang, J., Song, L., Wang, Y., Liu, C., Zhang, L., Zhu, S., Liu, S. and Duan, L., 2019. Beneficial effect of butyrate-producing Lachnospiraceae on stress-induced visceral hypersensitivity in rats. Journal of Gastroenterology and Hepatology 8: 1368-1376. https://doi.org/10.1111/jgh.14536

Zheng, J., Wittouck, S., Salvetti, E., Franz, C.M.A.P., Harris, H.M.B., Mattarelli, P., O'Toole, P.W., Pot, B., Vandamme, P., Walter, J., Watanabe, K., Wuyts, S., Felis, G.E., Gänzle, M.G. and Lebeer, S., 2020. A taxonomic note on the genus Lactobacillus: description of 23 novel genera, emended description of the genus Lactobacillus Beijerinck 1901, and union of Lactobacillaceae and Leuconostocaceae. International Journal of Systematic and Evolutionary Microbiology 70: 2782-2858. https://doi.org/10.1099/ ijsem.0.004107

Zheng, P., Zeng, B., Zhou, C., Liu, M., Fang, Z., Xu, X., Zeng, L., Chen, J., Fan, S., Du, X., Zhang, X., Yang, D., Yang, Y., Meng, H., Li, W., Melgiri, N.D., Licinio, J., Wei, H. and Xie, P., 2016. Gut microbiome remodeling induces depressive-like behaviors through a pathway mediated by the host's metabolism. Molecular Psychiatry 21: 786796. https://doi.org/10.1038/mp.2016.44 